\title{
IMPROVING LINEAR SPECTRAL UNMIXING THROUGH LOCAL ENDMEMBER DETECTION
}

\author{
R. Ramak ${ }^{\text {a }}$, M. J. Valadan Zouj ${ }^{\text {a }}$, B. Mojaradi ${ }^{\text {b }}$ \\ ${ }^{a}$ KNTU, Geomatics Engineering Faculty, 1996715433 Mirdamad and Valiasr ,Tehran, Iran - razieh.ramak@ gmail.com \\ Valadanzouj@kntu.ac.ir \\ b IUST, Civil Engineering Faculty, 1684613114 Narmak ,Tehran, Iran - Mojaradi@iust.ac.ir
}

KEY WORDS: Hyperspectral Data Set, Local Linear Spectral Unmixing (LLSU), Maximum Likelihood Classification (MLC)

\begin{abstract}
:
There are a considerable number of mixed pixels in remotely sensed images. Different sub-pixel analyses have been recently developed correspondingly. A well-known method is linear spectral unmixing which obtains an abundance of each endmember in a given pixel. This model assumes that each pixel is a linear combination of all endmembers in a scene. This assumption is not correct since each pixel can only be a composition of some surrounding endmembers. Even though, a fully mathematical technique is used for spectral analysis, the output of the model may not represent the physical nature of the objects over the pixel under test. In this regard, this paper proposes a Local Linear Spectral Unmixing which is based on neighbor pixels classes. Having classified the image, using a supervised classifier, it is scanned through a window of an appropriate size. For each pixel at the center of the window, the endmember matrix is formed only based on the majority classes existed in the window. Then the amount of each one is calculated. The LLSU method was evaluated on an AVIRIS data set collected from an agricultural area of northern Indiana. The results of the proposed method demonstrate a significant improvement in comparison with the LSU results. Moreover, due to the dimension reduction of the endmember matrix in this method, the computation time of the LLSU speeds up by three to eight times compared to the conventional Linear Spectral Unmixing method. As a result, the proposed method is efficient over the spectral unmixing tasks.
\end{abstract}

\section{INTRODUCTION}

One of the most common techniques for extracting information from hyperspectral images is classification which replaces the value of pixels in primary images with the label of classes to generate thematic maps. There are different techniques for classifying these images from which maximum likelihood classification (MLC) is one of the most important and commonly used techniques ( $\mathrm{Li}, 2007)$. ML hard classification is assigning each pixel to only one specific class according to its spectral characteristics (Richards, 1993a). However, satellite multi/ hyperspectral images are generally composed of mixed pixels, so that there might be several categories (corresponding with several classes) in one pixel (Keshava, 2002a)- (Keshava, 2005b), (Harsanyi, 1994a), (Velez-Reyes, 2003), (Shaw, 2002). Versus unmixing method like LSU allows the assignment of one pixel to multiple classes. LSU model is a technique to estimate proportion of each class in every pixel. Hence it will bring us more additional information (Keshava, 2002a), (Harsanyi, 1994a), (Velez-Reyes, 2003), (Shaw, 2002), (Smith, 1994), (Schowengerdt, 1997), (Adams, 1986). First assumption of this model is that every pixel is a linear combination of all spectra (classes) in a hyperspectral scene. This model can be written as:

$$
X=\sum_{i=1}^{c} a_{i} s_{i}+w=S A+w
$$

where

$$
c=\text { the number of endmembers }
$$$$
w=\text { error term }
$$

$$
\begin{aligned}
& a_{i}=\text { the proportion of endmember } \mathrm{i} \text { in pixel } \mathrm{X} \\
& s_{i}=\text { the ith endmember }
\end{aligned}
$$

So $\mathrm{S}$ is the endmember matrix and $\mathrm{A}$ is the abundance vector. The proportion of this model has to satisfy two constraints in the following equations (Nascimento, 2005a), (Manolaskis, 2003a), (Zare, 2010a), (Chang, 2000a), (Heinz, 2001a), (Huck, 2010a):

$$
\begin{gathered}
a_{i} \geq 0 \quad i=1,2, \ldots, C \\
\sum_{i=1}^{c} a_{i}=1
\end{gathered}
$$

It is noticeable that the probability of having all classes of the scene in one pixel is almost equal to zero, and every mixed pixel probably is a combination of surrounding spectra. In other words, assuming all classes gathered in one pixel is not based on reality. This is rooted in the fact that a mathematical model is used to estimate classes in a physical surface, and making such assumption may lead to huge errors in the results. By taking surrounding pixels' spectra into account and the output of another classifier, we can improve the results of LSU for identifying probable classes in a pixel.

There are different solutions to improve conventional LSU techniques using spectral information in surrounding pixels. These solutions apply supervised and unsupervised methods to estimate endmember's spectra. For instance, sub-pixel mapping algorithm proposed by Yong Ge et al. (Yong, 2009a), partitions an image into $3 \times 3$ blocks and estimates proportion of each endmember in a mixed pixel and neighbor pixel using soft classification. This technique is used to prepare sub-information 
for spatial distribution of the classes in mixed pixels. Senya Kiyasu et al. (Kiyasu, 2006), offered an adaptive sub-pixel estimation technique that applies the same solution to partition the surface of the image into $5 \times 5$ blocks, and then estimates the number of classes and spectrum for each block through an unsupervised method. Wataru Murakami et al. (Murakami, 2007), also applied a semi-supervised method to estimate the proportion of each class in a pixel of a remotely sensed image. In these methods, first, a small amount of training data will be prepared to be used in order to identify pure pixels in the image. Afterward, spectrum of endmembers for each mixed pixel will be estimated using neighbour pure pixels through an adaptive method. Then, each endmember's proportion in a mixed pixel will be estimated based on the endmembers' spectrum. The method used in this context is a Local Linear Spectral Unmixing (LLSU) technique which uses the label of neighbour pixels generated by MLC and a window with odd dimensions to unmix the central pixel. For further illustration, refer to the data shown in Figure 1. This figure depicts a part of hyperspectral data set in a window including three categories (endmembers) $\mathrm{A}, \mathrm{B}$, and $\mathrm{C}$. in which the central pixel (under-test pixel) was generated from two endmembers: A and B. In this model, endmember matrix is only composed of three columns (corresponding to the three classes) and the pixel spectral analysis is only based on these three classes. The LLSU representation was able to appropriately partition the central pixel into two regions and find proportion of each endmember accurately.

In the following, Section II presents the local linear spectral unmixing model. Experiment and results are shown in Section III. Conclusions and a discussion on future work are given in Section IV.

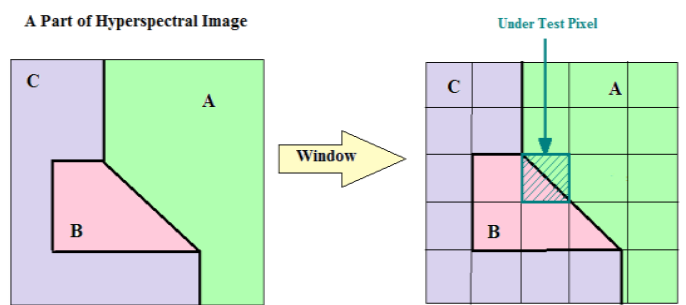

Figure 1. A schematic image of a part of hyperspectral data set in a $5 \times 5$ window including three classes

\section{DEVELOPING SPECTRAL UNMIXING MODEL}

Since most of the pixels in hyperspectral data are mixed, just the methods are successful that deal with these images according to their nature. For this reason, we decided to use unmixing methods instead of other conventional classifiers (e.g. MLC, SVM, and SVDD) for hard classification. On the other hand, the conventional unmixing methods like LSU suffer from aforementioned problems (i.e. neglecting of modeling the real imaging situation and encompassing all endmembers in a given pixel in its unmixing model). To provide an efficient method, we propose to use not only LSU for hard classification but also a remedy for the shortcoming of this method through utilizing LSU locally. Hence, we proposed a method focusing on endmember matrix determination that presents a real situation of the imaging scene for a given pixel by its surrounding endmembers. The scheme of the proposed method is shown in Figure 2. , includes the following steps:

Atmospheric correction: in this stage, to improve accuracy, atmospheric correction is performed on the achieved radiance image to produce reflectance data cube.

Dimension reduction: the informative bands are selected based on the wavelet transform to produce relevant bands for making use of MLC and to test the effect of dimension reduction on the spectral unmixing results.

Supervised classification: in this stage, to obtain the label of each pixel, the supervised classification using MLC was done on those bands that accomplished by SBFS method.

Local endmember selection: in this stage, for a given pixel, a window is determined with the size of $\mathrm{N}$ then the majority classes are accounted in that window.

LLSU: in this stage, LSU is performed based on the endmember matrix obtained for a given pixel in the third stage. Hardening process: in this stage, the fraction maps obtained from the previous stage is converted to the hard classified image through the winner takes all method.

\section{EXPERIMENTAL RESULTS}

To assess the performance of the proposed method an AVIRIS Indiana Pine (Online), hyperspectral data set with the size of $145 \times 145$ pixels is considered. This data set has 220 spectral bands in the range of $0.4-2.5 \mathrm{~nm}$ with a spatial resolution of $20 \mathrm{~m}$ and has 16 classes. It contains approximately two-thirds agricultural land and one-third forest and other elements. 20 water absorption channels (numbered 104-108, 150-163, and 220) were removed from the original image. In addition, 15 noisy channels, numbered 1-3, 103, 109-112, 148-149, 164 165 , and 217-219 as observed from visual inspection [21], were also removed, resulting in a total of 185 channels. The Airborne Visible/Infrared Imaging Spectrometer (AVIRIS) Indian Pines hyperspectral data set and its ground truth map are also shown in Figure 3. Since some of the classes are too small to retain enough disjoint samples for training and testing, three classes, Alphalpha, Grass/pasture-mowed and Oats (numbered 1,7, and 9 respectively) were leaved, retaining thirteen classes for the experiments including Corn-notill, Corn-min, Corn, Grass/Pasture, Grass/Trees, Hay-windrowed, Soybeans-no till, Soybeans-min, Soybeans-clean, Wheat, Woods, Bldg-grassgreen-drives and Stone-steel-towers (numbered 2-6, 8, and 1016 respectively). To illustrate the performance of the algorithm from all accessible samples $30 \%$ of samples are used as training data and the remaining $70 \%$ are used as test data.

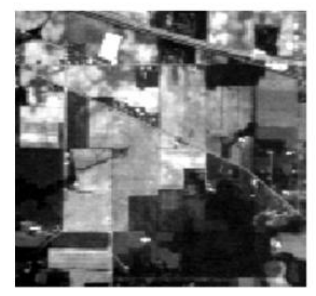

(a)

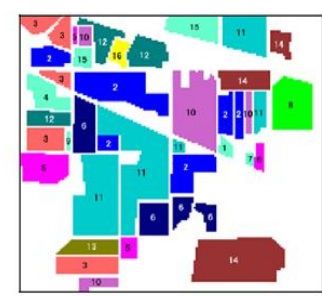

(b) 
Figure 3. (a) The AVIRIS Indian Pine data set. (b) Ground truth of the AVIRIS Indian Pine data set.

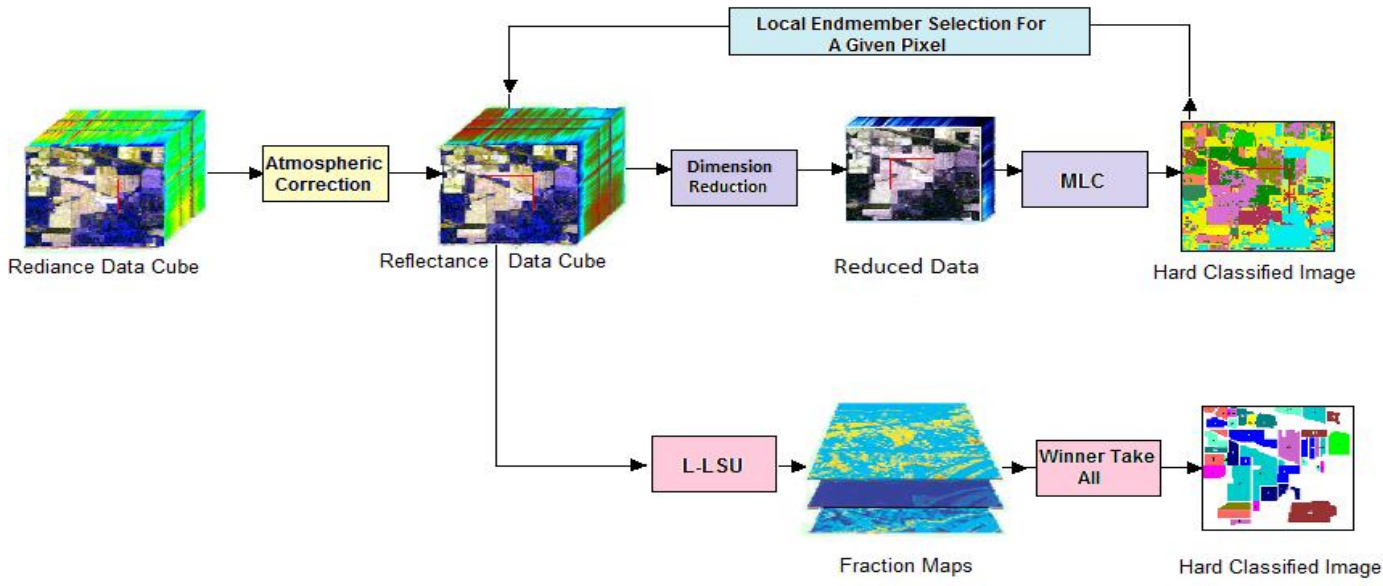

Figure. 2. The end-to-end Local Linear Spectral Unmixing algorithm (LLSU)

\subsection{Dimension Reduction}

Among available methods for dimension reduction that are described in the literature (Fukunaga, 1990), (Green, 1988a), (Ifarraguerri, 2002a), (Hsu, 2000), this study uses automatic wavelet transform because it preserves the distinction between spectral signatures and those computed in an automatic fashion. This is due to the intrinsic properties of Wavelet Transform that preserve high and low frequency feature therefore preserving peaks and valleys found in typical spectra; hence it can be useful for our purpose. Here wavelet transform is applied in three level of decomposition. First, features are reduced from 185 to 193 , at the second level from 93 to 47 and at the third level from 47 to 24 channels.

\subsection{Experiment 1: Conventional hard classification and unmixing method}

The goal of this experiment is Performing MLC to achieve the label of each pixel (or a thematic map), studying the effect of the dimension reduction on the LSU results, and comparing with the LLSU. MLC applied on the reduced dimension data set with 24 bands (from wavelet transform at third level of decomposition). Overall Accuracy (OA) term is used to accuracy assessment. The obtained result is presented in the first row of Table 1. Moreover, the LSU is performed in full dimension and reduced data using NCLS and FCLS algorithms (Chang, 2000a), (Heinz, 2001a). Since the soft ground truth data was not available for this data set, after decomposition for both global and local LSU, fraction maps were transferred to a hard map. So that, the class with the maximum abundance of each pixel will be considered as a wining class and the whole pixel will be labeled with this. Results of this test are shown in the second row of Table 1. As demonstrated in Table 1. the best $\mathrm{OA}$ is equal to $\% 75.37$, which occurs when noisy and water absorption channels are removed from the data set.

\subsection{Experiment 2: Performing LLSU algorithm}

3.3.1 Case 1: In this experiment, LLSU is carried out using two windows of $5 \times 5$ and $7 \times 7$ sizes in the original feature space and reduced dimension achieved by wavelet transform. In this case, the best OA occurred where the 185 bands and window of sizes $5 \times 5$ are used. Compared to the LSU, the obtained result through the LLSU demonstrates $6 \%$ improvement in terms of OA. In contrast, the MLC has the OA of about $2 \%$ more than the LLSU. To overcome the shortcoming of the proposed method we motivated to tackle it using majority classes inside the windows on the thematic map.

3.3.2 Case 2: In this case to improve OA, endmembers with less than 3 pixels in each window are omitted. This means that only classes with high abundance will remain for determining endmember matrix. Then, all steps mentioned in case 1 will be repeated. The results of this experiment for case 1 and 2 called LLSU (1) and LLSU (2) are shown in Table 1. respectively. In this case, the highest accuracy achieved when the window with the size of $5 \times 5$ and 185 bands are used. The OA is equal to $86.40 \%$, which shows up to $11.03 \%$ improvement compared to the conventional LSU method. Furthermore, compared to MLC this superiority is demonstrated by almost $3 \%$ improvement.

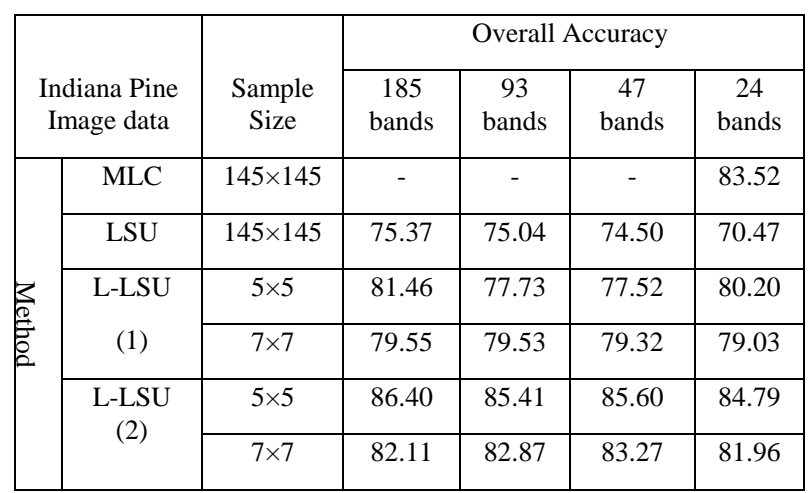


Table 1. Results of MLC, LSU and LLSU methods in case 1 and 2

\section{CONCLUSION AND FUTURE WORK}

A method to improve the LSU based on the surrounding information was presented in this study. First, the maximum likelihood algorithm was used to assign labels to classes. Using the information provided by the algorithm for each small window, the proportion of each class will be determined for each pixel. The obtained results showed that dimension reduction by the wavelet transform did not lead to the improvement of spectral unmixing results. Therefore, wavelet transform is not a proper method to reduce dimensions for improving the LSU results. In fact, presence of all bands, for conducting spectral unmixing, led to positive results. Of course, noisy bands need to be removed in the preprocessing stage. In addition, limiting endmember matrix to the neighbour endmembers of each pixel resulted in an improvement. It is noticeable that, conducting local linear spectral unmixing process, speed up the calculation. Since soft ground truth data for this data set was not available, it is not possible to make definite conclusion about the improving estimation of the fraction maps. However, increasing the OA after transforming soft classification results to hard one implies that the improvement in the estimation of the sub-pixel may be occurred. Although the proposed method is performed in many steps which increase the total time of the calculations, the significant improvement (11\% over the LSU method and 3\% over maximum likelihood classification) is remarkable.

Comparison between the accuracy of this method and the other methods mentioned in part 1 , is the issue of the future study. Also, it can be the possibility to achieve better results by other methods for selecting features. Hence, the effect assessment of the band selection through supervised method on LLSU will study in future.

\section{REFERENCES}

Adams, J. B. and Smith, M. O., 1986. "Spectral mixture modeling: A new analysis of rock and soil types at the Viking lander 1 suite," J. Geophys. Res., vol. 91, No. B8, pp. 80988112.

Chang, C-I., and Heinz, D.C., 2000a. "Constrained Subpixel Target Detection for Remotely Sensed Imagery" IEEE Trans. Geosci. Remote Sens., vol. 38, No. 3.

Fukunaga, K., 1990. Introduction to Statistical Pattern Recognition, 2nd ed. New York: Academic.

Green, A., Berman, M., Switzer, P., and Craig, M. D., 1988a . "A transformation for ordering multispectral data in terms of image quality with implications for noise removal," IEEE Trans. Geosci. Remote Sens., vol. 26, No. 1, pp. 65-74.

Harsanyi, J.C., Chang, C.I., 1994a. "Hyperspectral Image Classification and Dimensionality Reduction: An Orthogonal
Subspace Projection Approach,” IEEE Trans. Geosci. Remote Sens., vol. 32, No. 4.

Heinz , D.C., and Chang, C-I., 2001a . " Fully Constrained Least Squares Linear Spectral Mixture Analysis Method for Material Quantification in Hyperspectral Imagery," IEEE Trans. Geosci. Remote Sens., vol. 39, No. 3.

Huck, A., Guillaume, M., and Blanc-Talon, J., 2010a. "Minimum Dispersion Constrained Nonnegative Matrix Factorization to Unmix Hyperspectral Data," IEEE Trans. Geosci. Remote Sens., vol. 48, No. 6.

Hsu, P.H., and Tseng, Y.H., 2000. "Wavelet Based Analysis of Hyperspectral Data for Detecting Spectral Features,"

International Archives of Photogrametry and Remote Sensing .Vol.XXXIII, Supplement B7.

Ifarraguerri, A., and Chang, C.I., 2000a. "Unsupervised Hyperspectral Image Analysis With Projection Pursuit," IEEE Geosci.Remote Sensing, Vol.38, No. 6.

Keshava, N., and Mustard, J. F., 2002a. "Spectral unmixing," IEEE Signal Process.Mag., vol. 19, No. 1, pp. 44-57.

Keshava, N., 2003b. "A Survey of Spectral Unmixing Algorithms", Lincoln Laboratory Journal, vol. 14, No. 1.

Kiyasu, S., Terashima, K., Hotta, S., and Miyahara, S., 2006. "Adaptive subpixel estimation of land cover in a remotely sensed multispectral image," SICE-ICASE International Joint Conference,Oct. 18-21 in Bexco, Busan, Korea.

Li, B., Chi, M., Fan, J., Xue, X., 2007, "Support Cluster Machine" in proc. $24^{\text {th }}$ Int. Conf. Mach. Learn., Corvallis, OR,pp. 505-512.

Manolakis, D., Marden, D., and Shaw, G. A., 2003a. "Hyperspectral image processing for automatic target detection applications,” Lincoln Lab. J., vol. 14, No. 1, pp. 79-116.

Murakami, W., Nakama, R., Kiyasu, S., and Miyahara, S., 2007. "subpixel estimation of land cover in a remotely sensed image using spectral information of surrounding pixels," SICE Annual Conference,Sept. 17-20, in Kagawa University, Japan.

Nascimento, J. M. P., and Bioucas-Dias, J. M., 2005a. "Does independent component analysis play a role in unmixing hyperspectral data," IEEE Trans.Geosci. Remote Sens., vol. 43, No. 1, pp. 175-187.

[Online].Available:ftp://ftp.ecn.purdue.edu/biehl/MultiSpec/

Richards, J.A., 1993a. Remote Sensing Digital Image Analysis: An Introduction, 2nd Ed. New York: Springer-Verlargg.

Shaw, G., and Manolakis, D., 2002a. "Signal Processing for Hyperspectral Image Exploitation," IEEE Signal Processing Magzin, vol. 19, No.1, pp. 12-16.

Smith, M. O., Adams, J. B., and Sabol, D. E., 1994. "Spectral mixture analysis New strategies for the analysis of multispectral data," in Image Spectroscopy-A Tool for Environmental Observations, J. Hill and J.Mergier, Eds. Brussels, Belgium: ECSC, EEC, EAEC, pp.125-143. 
Schowengerdt, R. A., 1997. Remote Sensing: Models and Methods for Image Processing, 2nd ed. New York: Academic, pp. 470-471.

Vélez-Reyes, M., Puetz, A., Hoke, P., Lockwood, R. B., and Rosario, S., 2003 "Iterative Algorithms for Unmixing of Hyperspectral Imagery," Conference on Algorithms and Technologies for Multispectral, Hyperspectral, and Ultraspectral Imagery IX, Vol. 5093, pp. 418-429.

Varshney, P. K., and Arora, M. K., 2004a, Advanced Image Processing Techniques for Remotely Sensed Hyperspectral Data. Berlin, Germany: Springer-Verlag.

Yong, Ge., Sanping, Li., and Chris Lakhan, V., 2009a. "Development and testing of a subpixel mapping Algorithm", IEEE Transactions on geosciences and remote sensing, vol 47, No. 7.

Zare, A., and Gader, P., 2010a. "PCE: Piecewise Convex Endmember Detection", IEEE Transactions on geosciences and remote sensing, vol. 48, No. 6 . 